\title{
Thermodynamic Inequalities for Percolation
}

\author{
R. Durrett* and B. Nguyen \\ Department of Mathematics, U.C.L.A., Los Angeles, CA 90024, USA
}

\begin{abstract}
In this paper we describe the percolation analogues of the Gibbs and Helmholtz potentials and use these quantities to prove some general inequalities concerning the critical exponents of percolation processes.
\end{abstract}

\section{Introduction}

The main results in this paper and the reason for interest in them can be explained in a few words: Scaling theory (see e.g. [11, Sect. 4]) predicts that the critical exponents of a percolation process should satisfy

$$
\begin{gathered}
\gamma^{\prime}=\beta(\delta-1), \\
\beta(\delta+1)=2-\alpha^{\prime}, \\
\Delta=\beta+\gamma,
\end{gathered}
$$

and we have been able to show

$$
\begin{gathered}
\gamma^{\prime} \geqq \beta(\delta-1), \\
\beta(\delta+1) \geqq 2-\alpha_{2}^{\prime}, \\
\Delta_{2} \geqq 2, \Delta_{2}^{\prime} \geqq\left(\beta+\gamma^{\prime}\right) \wedge 2 .
\end{gathered}
$$

To make these inequalities meaningful we will have to give a number of definitions.

The first thing we have to describe is the class of models under consideration. The basic ingredients for a percolation process are

(i) a set of sites $S$,

(ii) a set of bonds $B C S \times S$,

(iii) a collection of random variables $\eta(x) x \in S$ which take values in $\{0,1\}$,

(iv) a family $P_{p} 0 \leqq p \leqq 1$ of probability measures so that under $P_{p}$ the $\eta$ 's are independent and have $P_{p}(\eta(x)=1)=p$.

* Partially supported by NSF grant MCS 83-00836, this author is an AMS Postdoctoral Research Fellow 1984-1986

Address after July 15, 1985: Department of Mathematics, Cornell University, Ithaca, NY 14853, USA 
The following examples should illustrate the range of possibilities and help explain the definitions which will follow.

1. Site percolation in $Z^{d}$

$$
S=Z^{d} \quad B=\{(x, y):|x-y|=1\} .
$$

2. Oriented site percolation in $Z^{d}$

$$
S=Z^{d} \quad B=\{(x, y): x \leqq y \quad|x-y|=1\} .
$$

3. Discrete time contact process in $Z^{d-1}$

$$
S=Z^{d} \quad B=\left\{((x, n),(y, n+1)): x, y \in Z^{d-1} \quad|x-y| \leqq 1\right\} .
$$

4. Bond percolation in $Z^{d}$

$$
S=\{(x, y):|x-y|=1\},
$$

$$
B=\{((u, v),(x, y)):(u, v) \text { and }(x, y) \text { have a common endpoint }\} .
$$

5. Percolation on the binary tree

$$
\begin{gathered}
S=\text { finite strings of } 0^{\prime} s \text { and } 1^{\prime} s, \\
B=\{(x, x 0),(x, x 1): x \in S\} .
\end{gathered}
$$

We think of the $x \in S$ as points in space, which are open if $\eta(x)=1$, and closed if $\eta(x)=0$, and we think of the set of bonds $B$ as indicating which points are adjacent. With these interpretations in mind we say that $y$ can be reached from $x$ and write $x \rightarrow y$ if there is a sequence of sites $x_{0}, \ldots, x_{m}$ with $x_{0}=x, x_{m}=y$ which has (a) $\left(x_{i-1}, x_{i}\right) \in B$ for all $1 \leqq i \leqq m$ and (b) $\eta\left(x_{i}\right)=1$ for all $0 \leqq i \leqq M$.

Let $C_{0}=\{x: 0 \rightarrow x\}=$ the set of points which can be reached from 0 and let $\left|C_{0}\right|$ $=$ the number of points in $C_{0}$. The main object of study in percolation is the distribution of $\left|C_{0}\right|$ and in particular what is the probability of $\Omega_{\infty}=\left\{\left|C_{0}\right|=\infty\right\}=$ "percolation" occurs. It is easy to show that if we throw out the trivial case $S=Z^{1}$, then in the examples above, $p_{c}=\inf \left\{p: P_{p}\left(\Omega_{\infty}\right)>0\right\}$ is in $(0,1)$ but it has turned out to be very difficult to answer basic questions about $p_{c}$ and the behavior of the process at the critical value:

(i) What is $p_{c}$ ?

(ii) Is $P_{p}\left(\Omega_{\infty}\right)=0$ when $p=p_{c}$ ?

For Examples 1-4 above these are open problems in $d \geqq 2$ and only known in $d=2$ for (i) Example 4, (ii) Examples 1 and 4, see [18] for details.

Given the state of our knowledge concerning $p_{c}$ it may come as somewhat of a surprise that it has been possible to prove general results concerning the behavior of percolation quantities as $p \rightarrow p_{c}$ without knowing what $p_{c}$ is! To state the results which have obtained and explain our contribution we have to introduce the critical exponents which appear in (1)-(3). To save time we will simply define them in one fell swoop and then make some comments to explain the definitions.

To shorten the definitions of our critical exponents we will write

$$
f(p) \approx\left(p-p_{c}\right)^{\lambda} \text { as } p \downarrow p_{c}
$$

as an abbreviation for: $f(p)=\left(p-p_{c}\right)^{\lambda} L\left(p-p_{c}\right)$, where $L$ is a slowly varying function, i.e.

$$
\lim _{t \downarrow 0} L(t y) / L(t)=1 \text { for all } y \in(0, \infty) \text {. }
$$


This is a little stronger than the usual definition (see e.g. [22, p. 32]) but (a) it is expected that most examples will have this type of behavior and (b) this definition allows us to use familiar Tauberian theorems rather than having to invent new ones.

With the $\approx$ notation introduced, we can quickly define the Greek letters which appear in our inequalities:

$$
\begin{array}{ll}
P_{p}\left(\Omega_{\infty}\right) \approx\left(p-p_{c}\right)^{\beta}, & \text { as } \quad p \downarrow p_{c}, \\
E_{p}\left|C_{0}\right| \approx\left(p-p_{c}\right)^{-\gamma}, & \text { as } \quad p \uparrow p_{c}, \\
E_{p}\left(\left|C_{0}\right| ;\left|C_{0}\right|<\infty\right) \approx\left(p-p_{c}\right)^{-\gamma^{\prime}}, & \text { as } \quad p \downarrow p_{c}, \\
E_{p}\left|C_{0}\right|^{k} / E_{p}\left|C_{0}\right|^{k-1} \approx\left|p-p_{c}\right|^{-\Delta_{k}}, & \text { as } \quad p \uparrow p_{c}, \\
\frac{\partial^{2}}{\partial p^{2}} E_{p}\left(\left|C_{0}\right|^{-1} ;\left|C_{0}\right| \geqq 1\right) \approx\left(p-p_{c}\right)^{-\alpha_{2}^{\prime}}, & \text { as } \quad p \downarrow p_{c}, \\
\frac{\partial^{3}}{\partial p^{3}} E_{p}\left(\left|C_{0}\right|^{-1} ;\left|C_{0}\right| \geqq 1\right) \approx\left(p-p_{c}\right)^{-\alpha_{3}^{\prime}-1}, & \text { as } \quad p \downarrow p_{c}, \\
P_{p_{c}}\left(\left|C_{0}\right|>n\right) \approx n^{-1 / \delta}, & \text { as } n \rightarrow \infty,
\end{array}
$$

where in the last line have we used an obvious extension of the definition of $\approx$.

The first three definitions and the last one are self-explanatory (or if they are not see [9]). If you compare the second and third and look at the fourth, you see that we could have defined $\Delta_{k}^{\prime}$ by considering the behavior of $E_{p}\left(\left|C_{0}\right|^{k} ;\left|C_{0}\right|<\infty\right)$ as $p \downarrow p_{c}$, and a quick glance at the table confirms that with the exception of $\beta$ (which only makes sense for $p \downarrow p_{c}$ ), primes are used to indicate approach from $p>p_{c}$. We use the same Greek letters for $p \uparrow p_{c}$ and $p \downarrow p_{c}$ because "conventional wisdom" tells us that the exponents coming from the two directions of approach will be equal. This is true in Example 5 (see [9]) but it is far from obvious why this should be true in general.

Last but not least we have the two definitions of $\alpha$. The first one is the naive analogue of the Ising model definition and not coincidentally the one that we can prove something about. The second definition is probably the right definition. Kesten has shown (see [18, Theorem 9.4]) that for two dimensional percolation problems which are part of a matching pair (e.g. Examples 1 and 4), then $E_{p}\left(\left|C_{0}\right|^{-1} ;\left|C_{0}\right| \geqq 1\right)$ is $C^{2}$ on $[0,1]$ (and hence $\alpha_{2}^{\prime}=0$ ) and his calculations suggest that $\partial^{3} F / \partial p^{3} \rightarrow \infty$. The reader should note that thanks to the -1 in the definition of $\alpha_{3}^{\prime}$ we have $\alpha_{2}^{\prime} \geqq \alpha_{3}^{\prime}$ with equality if $\alpha_{2}^{\prime}>0$, i.e. $\alpha_{2}^{\prime}=\left(\alpha_{3}^{\prime}\right)^{+}$, so if you want, (2) can be rewritten as $\beta(1+\delta) \geqq 2-\left(\alpha_{3}^{\prime}\right)^{+}$.

With the definitions of the critical exponents completed we have finally introduced all the variables which appear in our inequalities. The last step in making (1) - (3) meaningful statements is to state what we must assume about percolation for our proofs to work:

(i) $K=\sup _{x \in S}|\{y:(x, y) \in B\}|<\infty$,

(ii) $p_{c} \in(0,1)$,

(iii) $P_{p}\left(\Omega_{\infty}\right)=0$ when $p=p_{c}$. 
Of these the first two are very natural and are known to hold for all our examples (in $d \geqq 2$ ). The third is a little undesirable but the first inequality is trivial if $\beta=0$ and our proof of the second breaks down if $P_{p}\left(\Omega_{\infty}\right) \rightarrow 0$ as $p \downarrow p_{c}$.

The rest of the paper is devoted to proving (1)-(3) and explaining why they hold. In Sect. 2 we (re)define the free energy $F(p, h)$, discuss some of its properties and prove (1). In Sect. 3 we show that although $F(p, h)$ is not always convex in $p$ (it is not in Example 5), if we pick $C$ large enough then $C\left(p-p_{c}\right)^{2}+F(p, h)$ is a convex function of $(p, h)$ near $\left(p_{c}, 0\right)$. In Sect. 4 we define the Helmholtz potential

$$
A(p, \theta)=\sup _{h>0}(\theta h-F(p, h))
$$

(a.k.a. conjugate convex function) and show that it looks enough like its Ising model analogue so that we can use Griffith's [15] argument to prove (2). Finally the proof of (3) is given in Sect. 5. It is a simple computation which is essentially independent of previous developments.

\section{The Gibbs Potential}

The first step in our investigation of relationships between critical exponents is to (re)define the percolation analogue of the free energy of a magnetic system or, to be precise -1 times the Gibbs potential (see [23, p. 29]). We let

$$
F(p, h)=h\left(1-\alpha_{0}(p)\right)+\sum_{n=1}^{\infty} n^{-1} \alpha_{n}(p) e^{-n h},
$$

where $\alpha_{n}(p)=P_{p}\left(\left|C_{0}\right|=n\right)$, and the sum here and below is over $1 \leqq n<\infty$. The second term in the expression above was first introduced by Kasteleyn and Fortuin (1969) to set up a correspondence between quantities for percolation and analogous quantities for magnetic systems which we will now describe.

If $G(T, H)$ is the Gibbs potential for a magnetic system at temperature $T$ and in an external field $H$, then the magnetization $M$ is defined by

$$
M(T, H)=-\left(\frac{\partial G}{\partial H}\right)_{T}
$$

(see [23, p. 34], the subscript $T$ indicates a derivative taken "at constant temperature"). If we differentiate $F(p, h)$ with respect to $h$, we get

$$
\frac{\partial F}{\partial h}(p, h)=1-\alpha_{0}(p)-\sum_{n=1}^{\infty} \alpha_{n}(p) e^{-n h},
$$

and when we set $h=0$ we get

$$
\frac{\partial F}{\partial h}(p, 0)=P_{p}\left(\left|C_{0}\right|=\infty\right) .
$$

[This is one reason for adding $h\left(1-\alpha_{0}(p)\right)$ to the old definition, another one will appear in Sect. 4.]

The last computation suggests that we should think of $h$ as the "external magnetic field" and $M(p, h) \equiv \partial F / \partial h(p, h)$ as being the "magnetization." For 
arguments below it is useful to note that these quantities have interpretations for percolation. Consider a new percolation process in which each site in $S$ is connected by a bond to a "ghost" site * and each of the bonds is closed with probability $e^{-h}$ and open with probability $1-e^{-h}$. If in the modified system $\left|C_{0}\right|=n$ then $* \notin C_{0}$ and hence no point in $C_{0}$ can be connected to $*$, so introducing some obvious notation for probabilities in the new system we have

$$
P_{p, h}\left(\left|C_{0}\right|=n\right)=\alpha_{n}(p) e^{-n h},
$$

and

$$
M(p, h)=P_{p, h}\left(\left|C_{0}\right|=\infty\right) .
$$

Having taken one derivative and found the percolation probability, the inevitable next step is to take another and see what we get

$$
\chi(p, h)=\frac{\partial^{2} F}{\partial h^{2}}(p, h)=\sum_{n=1}^{\infty} n \alpha_{n}(p) e^{-n h}=E_{p, h}\left(\left|C_{0}\right| ;\left|C_{0}\right|<\infty\right),
$$

i.e. the mean size of finite clusters in the modified system. We have used the letter $\chi$ above because the mean cluster size is the analogue of the (isothermal) susceptibility of magnetic systems which is obtained by differentiating the magnetic field $M$ with respect to $H$ (see [23, p. 35]). The reader should note that thanks to the exclusion of $n=\infty$ from the sum

$$
\chi(p, 0)=\lim _{h \downarrow 0} \chi(p, h)=E_{p}\left(\left|C_{0}\right| ;\left|C_{0}\right|<\infty\right) .
$$

The analogue of this quantity for the Ising model is

$$
\sum_{x}\langle\xi(0) \xi(x)\rangle_{+}-\langle\xi(0)\rangle_{+}\langle\xi(x)\rangle_{+},
$$

where $\langle f\rangle_{+}=\int f d \mu^{+}, \mu^{+}$being the Gibbs state which is the limit with + boundary conditions (for more about this quantity see [22] or [1]).

Having found how $M$ and $\chi$ (and hence $\beta$ and $\gamma$ ) are related to the free energy, the next step is to find $\delta$ and $\alpha_{2}^{\prime}$,

$$
M\left(p_{c}, h\right)-M\left(p_{c}, 0\right)=\sum_{n=0}^{\infty} \alpha_{n}\left(p_{c}\right)\left(1-e^{-h n}\right),
$$

and we have assumed $M\left(p_{c}, 0\right)=0$, so if $P_{p}\left(\left|C_{0}\right|>n\right) \approx n^{-1 / \delta}$ and $\delta>1$, it follows from a standard Tauberian theorem (see e.g. [12, Sect. 13]) that $M(p, h) \approx h^{1 / \delta}$.

On the other hand $F(p, 0)=E_{p}\left(\left|C_{0}\right|^{-1} ;\left|C_{0}\right| \geqq 1\right)$, so

$$
\frac{\partial^{2} F}{\partial p^{2}}(p, 0) \approx\left(p-p_{c}\right)^{-\alpha_{2}^{\prime}} \text { as } p \downarrow p_{c} \text {. }
$$

Comparing the last two formulas with the definition of $\beta$, and the two $\gamma$ 's

$$
\begin{gathered}
M(p, 0) \approx\left(p-p_{c}\right)^{\beta} \text { as } p \downarrow p_{c}, \\
\chi(p, 0)=\frac{\partial M}{\partial h}(p, 0) \approx \begin{cases}\left(p-p_{c}\right)^{-\gamma} & p \uparrow p_{c} \\
\left(p-p_{c}\right)^{-\gamma^{\prime}} & p \downarrow p_{c}\end{cases}
\end{gathered}
$$


shows that all the exponents are concerned with some aspect of the behavior of $M(p, h)$ near $\left(p_{c}, 0\right)$, and given this it should not be surprising that there are some relationships between the exponents which hold for all percolation processes. If one is optimistic one can hope that near $\left(p_{c}, 0\right)$ the "singular part" of the free energy can be written as

$$
F_{\text {sing }}(p, h) \approx\left|p-p_{c}\right|^{2-\alpha} \Phi\left(h /\left|p-p_{c}\right|^{\Delta}\right)
$$

(see [11, Formula (4.27)]) or what is almost the same is that the "equation of state" has the homogeneous form,

$$
h=M^{\delta} \Phi\left(\left(p-p_{c}\right) / M^{1 / \beta}\right)
$$

(see [3, p. 9]). From either one of these assumptions (see [11, Sect. 4.3] or [3, p. 9]) one can conclude that

$$
\gamma=\gamma^{\prime}=\beta(\delta-1) .
$$

(This is an example of "Scaling Theory" at work.)

These assumptions while probably true, are virtually impossible to verify in nontrivial examples and nontrivial to prove even in trivial examples (try it on the tree). In the light of these difficulties it is interesting to observe that by using simple properties of $M$ we can show that

$$
\gamma^{\prime} \geqq \beta(\delta-1) .
$$

Proof. The proof of this result is an almost word for word translation of Griffiths [15] proof of the corresponding result for the Ising model [see p. 1961, Formulas (23)-(25)].

$$
\frac{\partial M}{\partial h}(p, h)=\chi(p, h)=E_{p, h}\left(\left|C_{0}\right| ;\left|C_{0}\right|<\infty\right)
$$

is a decreasing function of $h$ for fixed $p$, so $h \rightarrow M(p, h)$ is concave and hence

$$
M(p, h) \leqq M(p, 0)+h \chi(p, 0) \text {. }
$$

Now $h \rightarrow M(p, h)$ is an increasing function for fixed $p$, and we have assumed that $M\left(p_{c}, 0\right)=0$ and $p_{c} \in(0,1)$, so if $p>p_{c}$ and near $p_{c}$ we can pick $h$ and $h^{\prime}$ so that

$$
\begin{gathered}
M(p, h)=2 M(p, 0), \\
M\left(p_{c}, h^{\prime}\right)=2 M(p, 0),
\end{gathered}
$$

and since $p \rightarrow M(p, h)$ is an increasing function we have $h^{\prime} \geqq h$. Combining the results in the last sentence with (2) gives

$$
\begin{gathered}
2 M(p, 0)=M(p, h) \leqq M(p, 0)+h \chi(p, 0), \\
M(p, 0) \leqq h \chi(p, 0) \leqq h^{\prime} \chi(p, 0) .
\end{gathered}
$$

Now the definitions of the critical exponents imply that as $p \downarrow p_{c}$

$$
\begin{gathered}
M(p, 0) \approx\left(p-p_{c}\right)^{\beta}, \\
\chi(p, 0) \approx\left(p-p_{c}\right)^{-\gamma^{\prime}}, \\
h^{\prime} \approx(2 M(p, 0))^{\delta} \approx\left(p-p_{c}\right)^{\beta \delta},
\end{gathered}
$$


so (4) implies [recall $x^{2}<x$ when $x \in(0,1)$,

$$
\beta \geqq-\gamma^{\prime}+\beta \delta,
$$

which is the desired inequality.

\section{Convexity of the Modified Free Energy}

In the last section we used the fact that $h \rightarrow M(p, h)$ is concave to prove a relationship between critical exponents. For magnetic systems (see [23, p. 36]) the Gibbs potential is a concave function of $(p, h)$ and this can be used to prove further inequalities between critical exponents. Based on this we might hope that our free energy $F$ would be a convex function of $(p, h)$ at least near $\left(p_{c}, 0\right)$. The derivatives with respect to $h$ have the right sign

$$
\begin{gathered}
\frac{\partial F}{\partial h}(p, h)=1-\alpha_{0}(p)-\sum_{1 \leqq n<\infty} \alpha_{n}(p) e^{-n h} \geqq 0, \\
\frac{\partial^{2} F}{\partial h^{2}}(p, h)=\sum_{1 \leqq n<\infty} n \alpha_{n}(p) e^{-n h} \geqq 0,
\end{gathered}
$$

but the derivatives with respect to $p$ are not. A little computation (and a number of checks and rechecks to make sure the signs are right) shows that for percolation on the binary tree (see Appendix for details)

$$
F(p, h)=h p+(1-p) B\left(p(1-p) e^{-h}\right),
$$

where

$$
B(x)=4 \int_{0}^{x}(1+\sqrt{1-4 y})^{-2} d y \quad x \leqq \frac{1}{4}
$$

so

$$
\frac{\partial^{2} F}{\partial p^{2}}(1 / 2,0)=-B^{\prime}(1 / 4)=-4 .
$$

The last example shows that convexity of $F$ does not hold in general. The next result provides a substitute for convexity which is still good enough for some purposes

If $C$ is sufficiently large,

$$
\hat{F}(p, h)=C\left(p-p_{c}\right)^{2}+F(p, h)
$$

is a convex function of $(p, h)$ near $\left(p_{c}, 0\right)$.

Proof. The first step is get a more explicit expression for $P_{p}\left(\left|C_{0}\right|=n\right)$. Let

$$
\partial C_{0}=\left\{y: y \notin C_{0} \text { and there is an } x \in C_{0} \text { with }(x, y) \in B\right\} .
$$

A little thought reveals

$$
P\left(\left|C_{0}\right|=n,\left|\partial C_{0}\right|=m\right)=\alpha_{n, m} p^{n}(1-p)^{m},
$$


where

$\alpha_{n, m}=$ the number of clusters containing 0 with size $n$ and boundary of size $m$.

With this notation we can write

$$
F(p, h)=h p+\sum_{m, n} n^{-1} \alpha_{n, m} p^{n}(1-p)^{m} e^{-n h}
$$

[since $\alpha_{0}(p)=1-p$ for site percolation] and differentiate to get

$$
\begin{aligned}
\frac{\partial F}{\partial p}= & h+\sum_{m, n} n^{-1} \alpha_{n, m} e^{-n h}\left(\frac{n}{p}-\frac{m}{1-p}\right) p^{n}(1-p)^{m} \\
\frac{\partial^{2} F}{\partial p^{2}}= & \sum_{m, n} n^{-1} \alpha_{n, m} e^{-n h} p^{n}(1-p)^{m}\left(\frac{n}{p}-\frac{m}{1-p}\right)^{2} \\
& -\sum_{m, n} n^{-1} \alpha_{n, m} e^{-n h} p^{n}(1-p)^{m}\left(\frac{n}{p^{2}}+\frac{m}{(1-\dot{p})^{2}}\right) .
\end{aligned}
$$

The first term in $\partial^{2} F / \partial p^{2}$ is $>0$, but the second is

$$
-E_{p, h}\left(\frac{1}{p^{2}}+\frac{\left|\partial C_{0}\right| /\left|C_{0}\right|}{(1-p)^{2}}\right)<0 \text {. }
$$

However we have supposed $|\{y:(x, y) \in B\}| \leqq K<\infty$ for all $x \in S$ and that $p_{c} \in(0,1)$, so we have $\left|\partial C_{0}\right| \leqq K\left|C_{0}\right|$, and it follows that near $\left(p_{c}, 0\right)$ we have

$$
0 \leqq E_{p, h}\left(\frac{1}{p^{2}}+\frac{\left|\partial C_{0}\right| /\left|C_{0}\right|}{(1-p)^{2}}\right) \leqq K^{\prime}
$$

where $K^{\prime} \in(0, \infty)$, so if we pick $C \geqq 2 K^{\prime}$, we will have

$$
\frac{\partial^{2} \hat{F}}{\partial p^{2}} \geqq K^{\prime}+\sum_{m, n}^{\infty} n^{-1} \alpha_{n, m} e^{-n h} p^{n}(1-p)^{m}\left(\frac{n}{p}-\frac{m}{1-p}\right)^{2}>0 .
$$

To finish the proof of convexity we need to show that

$$
\left(\frac{\partial^{2} \hat{F}}{\partial p \partial h}\right)^{2} \leqq \frac{\partial^{2} \hat{F}}{\partial h^{2}} \frac{\partial^{2} \hat{F}}{\partial p^{2}}
$$

To do this we observe

$$
\frac{\partial \hat{F}}{\partial h}=\frac{\partial F}{\partial h}=p-\sum_{m, n} \alpha_{n, m} p^{n}(1-p)^{m} e^{-n h},
$$

so

$$
\frac{\partial^{2} \hat{F}}{\partial p \partial h}=1-\sum_{m, n}\left(\frac{n}{p}-\frac{m}{1-p}\right) \alpha_{n, m} p^{n}(1-p)^{m} e^{-n h}
$$

and

$$
\frac{\partial^{2} \hat{F}}{\partial h^{2}}=\sum_{m, n} n \alpha_{n, m} p^{n}(1-p)^{m} e^{-n h}
$$


Now if we let $\alpha_{n, m}(p, h)=\alpha_{n, m} p^{n}(1-p)^{m} e^{-n h}$ we have

$$
\left|\frac{\partial^{2} \hat{F}}{\partial p \partial h}\right| \leqq 1+\left|\sum_{m, n}\left(\frac{n}{p}-\frac{m}{1-p}\right) \alpha_{n, m}(p, h)\right| .
$$

Squaring both sides leads to three terms on the right. To handle the last one we observe that the Cauchy-Schwarz inequality implies

$$
\begin{aligned}
\left(\sum_{m, n}\left(\frac{n}{p}-\frac{m}{1-p}\right) \alpha_{n, m}(p, h)\right)^{2} & \leqq\left(\sum_{m, n} n \alpha_{n, m}(p, h)\right)\left(\sum_{m, n} n^{-1}\left(\frac{n}{p}-\frac{m}{1-p}\right)^{2} \alpha_{n, m}(p, h)\right) \\
& \leqq\left(\frac{\partial^{2} \hat{F}}{\partial h^{2}}\right)\left(\frac{\partial \hat{F}^{2}}{\partial p^{2}}-K^{\prime}\right)
\end{aligned}
$$

by the choice of $C$ made above.

To complete the proof of (3) and hence of (1) it only remains to show that near $\left(p_{c}, 0\right)$ we have

$$
K^{\prime}\left(\frac{\partial^{2} \hat{F}}{\partial h^{2}}\right) \geqq 1+2\left|\sum_{m, n}\left(\frac{n}{p}-\frac{m}{1-p}\right) \alpha_{n, m}(p, h)\right|,
$$

but this is easy;

$$
\frac{\partial^{2} \hat{F}}{\partial h^{2}}=\sum_{m, n} n \alpha_{n, m}(p, h) \geqq \varepsilon\left|\sum_{m, n}\left(\frac{n}{p}-\frac{m}{1-p}\right) \alpha_{n, m}(p, h)\right|
$$

near $\left(p_{c}, 0\right)$ and $\partial^{2} \hat{F} / \partial h^{2}\left(p_{c}, 0\right)>0$, so (4) holds if $K^{\prime}$ is sufficiently large.

At first glance the conclusion in (1) probably appears worthless because we have obtained the convexity by force. The next argument should show that (1) is indeed useful. More evidence will be given in the next section.

The starting point of our derivation of Rushbrooke's inequality is the fact that the convexity of $\hat{F}$ implies

$$
\left(\frac{\partial^{2} \hat{F}}{\partial p \partial h}\right)^{2} \leqq\left(\frac{\partial^{2} \hat{F}}{\partial p^{2}}\right)\left(\frac{\partial^{2} \hat{F}}{\partial h^{2}}\right)
$$

[this was (3) above] and the definitions of the critical exponents imply that as $p \downarrow p_{c}$,

$$
\begin{gathered}
\frac{\partial^{2} \hat{F}}{\partial p^{2}}=C+\frac{\partial^{2} F}{\partial p^{2}} \approx\left(p-p_{c}\right)^{-\alpha_{2}^{\prime}}, \\
\frac{\partial^{2} \hat{F}}{\partial h^{2}}=\frac{\partial^{2} F}{\partial h^{2}} \approx\left(p-p_{c}\right)^{-\gamma^{\prime}},
\end{gathered}
$$

so if we strengthen the definition of $\beta$ to

$$
\frac{\partial^{2} \hat{F}}{\partial p \partial h}=\frac{\partial^{2} F}{\partial p \partial h}=\frac{\partial M}{\partial p} \approx\left(p-p_{c}\right)^{\beta-1} \quad \text { as } \quad p \downarrow p_{c},
$$

it follows that we have

$$
2(\beta-1) \geqq-\alpha_{2}^{\prime}-\gamma^{\prime}
$$

which is Rushbrooke's inequality. 
The argument above is from Griffiths [15]. In the next section we will show that with the usual definition of $\beta$ we have $\beta(1+\delta) \geqq 2-\alpha_{2}^{\prime}$. Combining that result with the inequality of Sect. 3 gives a proof of Rushbrooke's inequality which avoids the extra assumption about $\beta$.

\section{Helmholtz Potential}

In Sect. 2 we defined the Gibbs potential. This is just one of four thermodynamic state functions for magnetic systems (see [23, pp. 22, 23]). Another quantity which has been useful in studying the Ising model is the Helmholtz potential

$$
A(p, \theta)=\sup _{h \geqq 0} \theta h-F(p, h) .
$$

For fixed $p \theta \rightarrow A(p, \theta)$ is the convex function conjugate to the function

$$
h \rightarrow \begin{cases}F(p, h) & h \geqq 0 \\ \infty & h<0\end{cases}
$$

(or in other words its Legendre transform) and as such is convex in $\theta$ for fixed $p$ (see [24, p. 156]).

The definition of $A$ may not appear natural at first, but the following computation should suggest that it is a useful quantity for studying percolation. If $p>p_{c}$, then

$$
\frac{\partial F}{\partial h}(p, 0)=P\left(\Omega_{\infty}\right)>0,
$$

and $h \rightarrow F(p, h)$ is convex, so if $\theta<M(p, 0)$,

$$
\sup _{h \geqq 0} \theta h-F(p, h)=-F(p, 0),
$$

i.e. $h \rightarrow A(p, h)$ is constant for $0<h<M(p)$.

The last conclusion is reminiscent of pictures of the free energy for magnetic systems (see Fig. 1 and/or cf. [23, p. 37]) and for the Ising model (see [15, p. 1959]) and $\theta \rightarrow A(p, \theta)$ is convex, so one might think (as Wu [25, p. 122] claimed) that we have all the basic ingredients needed in a derivation of Griffith's inequality. Unfortunately the crucial property for Griffith's proof is the fact that $p \rightarrow A(p, \theta)$ is concave for fixed $\theta$ [see Formula (7) on p. 1960], and the results given for percolation on the tree in the last section show that this need not hold in general.

Faced with this difficulty the way out as it was in the last section is to look at the modified free energy

$$
\hat{F}(p, h)=C\left(p-p_{c}\right)^{2}+F(p, h)
$$

and look at its Legendre transform

$$
\hat{A}(p, \theta)=\sup _{h \geqq 0}(\theta h-\hat{F}(p, h))=-C\left(p-p_{c}\right)^{2}+A(p, \theta) .
$$

Since $F(p, h)$ is a convex function of $(p, h)$ it follows easily that we have

$$
p \rightarrow A(p, \theta) \text { is concave for each } \theta .
$$


Fig. 1

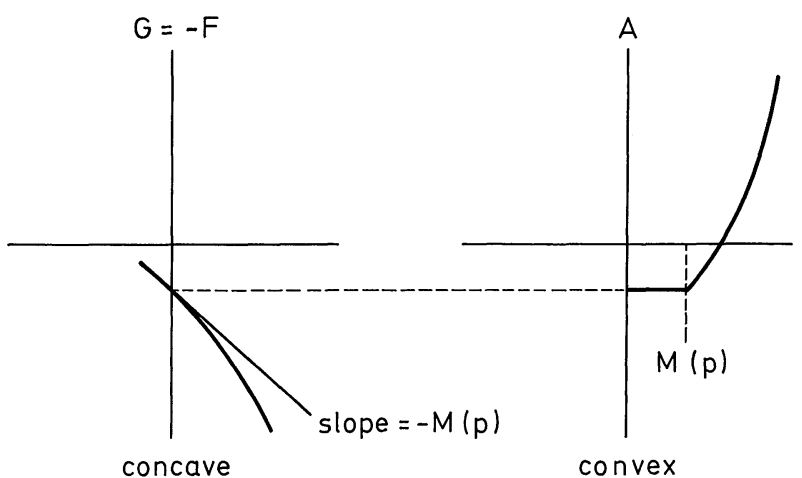

Proof. Let $p_{1}<p_{2}$, let $\varepsilon>0$, and pick $h_{1} h_{2}$ so that

$$
\theta h_{i}-F\left(p_{i}, h_{i}\right)>A\left(p_{i}, \theta\right)-\varepsilon .
$$

If $0 \leqq \lambda \leqq 1$

$$
\begin{aligned}
\lambda \hat{A} & \left(p_{1}, \theta\right)+(1-\lambda) \hat{A}\left(p_{2}, \theta\right)-\varepsilon \\
& \leqq \lambda\left(\theta h_{1}-\hat{F}\left(p_{1}, h_{1}\right)\right)+(1-\lambda)\left(\theta h_{2}-\hat{F}\left(p_{2}, h_{2}\right)\right) \\
& =\theta\left(\lambda h_{1}+(1-\lambda) h_{2}\right)-\left(\lambda \hat{F}\left(p_{1}, h_{1}\right)+(1-\lambda) \hat{F}\left(p_{2}, h_{2}\right)\right) \\
& \leqq \theta\left(\lambda h_{1}+(1-\lambda) h_{2}\right)-\hat{F}\left(\lambda p_{1}+(1-\lambda) p_{2}, \lambda h_{1}+(1-\lambda) h_{2}\right) \\
& \leqq \hat{A}\left(\lambda p_{1}+(1-\lambda) p_{2}, \theta\right),
\end{aligned}
$$

and since $\varepsilon$ is arbitrary, the result follows.

With (1) established we are now ready to follow Griffiths [15] argument with $A$ replaced by $\hat{A}$. The argument can be summarized in one picture:

Fig. 2

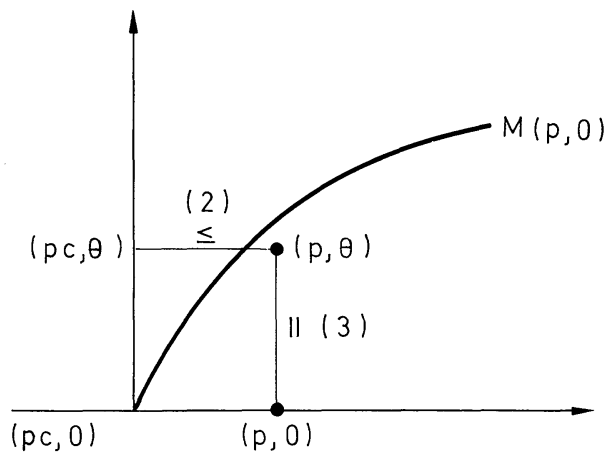

Let

$$
\hat{M}(p, h)=\frac{\partial \hat{F}}{\partial h}(p, h)=\frac{\partial F}{\partial h}(p, h), \quad \hat{S}(p, \theta)=\frac{\partial \hat{A}}{\partial p}(p, \theta) \neq \frac{\partial A}{\partial p}(p, \theta) .
$$

(The letter $S$ stands for entropy, see [23, pp. 22, 23].) 
As Griffiths [15] says "for both conceptual and computational purposes" it is convenient to introduce the starred quantities

$$
\begin{gathered}
\hat{A}^{*}(p, \theta)=\hat{A}(p, \theta)-\hat{A}\left(p_{c}, \theta\right)-\left(p-p_{c}\right) \hat{S}\left(p_{c}, 0\right), \\
\hat{S}^{*}(p, \theta)=\hat{S}(p, \theta)-\hat{S}\left(p_{c}, \theta\right)=\frac{\partial \hat{A}^{*}}{\partial p}(p, \theta) .
\end{gathered}
$$

Concavity of $\hat{A}^{*}$ implies

$$
\hat{A}^{*}\left(p_{c}, \theta\right) \leqq \hat{A}^{*}(p, \theta)+\left(p-p_{c}\right) \hat{S}^{*}(p, \theta),
$$

but if $\theta<M(p, 0)$,

$$
\begin{gathered}
\hat{A}(p, \theta)=\hat{A}(p, 0) \\
\hat{S}(p, \theta)=\frac{\partial \hat{A}}{\partial p}(p, \theta)=\frac{\partial \hat{A}}{\partial p}(p, 0)
\end{gathered}
$$

so we have

$$
\begin{gathered}
\hat{A}^{*}(p, \theta)=\hat{A}(p, 0)-\hat{A}\left(p_{c}, 0\right)-\left(p-p_{c}\right) \hat{S}\left(p_{c}, 0\right) \\
\hat{S}^{*}(p, \theta)=\hat{S}(p, 0)-\hat{S}\left(p_{c}, 0\right)
\end{gathered}
$$

and using (2)

$$
\hat{A}^{*}\left(p_{c}, \theta\right) \leqq \hat{A}(p, 0)-\hat{A}\left(p_{c}, 0\right)-\left(p-p_{c}\right) \hat{S}\left(p_{c}, 0\right)+\left(p-p_{c}\right)\left(\hat{S}(p, 0)-\hat{S}\left(p_{c}, 0\right)\right) .
$$

Now $\hat{A}(p, 0)=-\hat{F}(p, 0)=-F(p, 0)-C\left(p-p_{c}\right)^{2}$, so if

$$
\frac{\partial^{2} F}{\partial p^{2}} \approx\left(p-p_{c}\right)^{-\alpha_{2}^{\prime}} \quad \text { as } \quad p \downarrow p_{c},
$$

it follows that the right-hand side $\approx\left(p-p_{c}\right)^{2-\alpha_{2}^{\prime}}$ as $p \downarrow p_{c}$. To compute the behavior of the left-hand side we observe that

$$
\begin{aligned}
\hat{A}^{*}\left(p_{c}, \theta\right) & =\hat{A}\left(p_{c}, \theta\right)-\hat{A}\left(p_{c}, 0\right)=A\left(p_{c}, \theta\right)-A\left(p_{c}, 0\right) \\
& =\sup _{h \geqq 0}\left(\theta h-F\left(p_{c}, h\right)\right)+F\left(p_{c}, 0\right) .
\end{aligned}
$$

If $\theta>0$ the maximum of $\theta h-F\left(p_{c}, h\right)$ occurs at the $h(\theta)$ which is the solution of

$$
\frac{\partial F}{\partial h}\left(p_{c}, h\right)=\theta
$$

i.e. at the external magnetic field strength needed to produce a percolation probability $=\theta$, and we have assumed

$$
\frac{\partial F}{\partial h}\left(p_{c}, h\right) \approx h^{1 / \delta}
$$

as $h \rightarrow 0$ so $h(\theta) \approx \theta^{\delta}$. Now writing

$$
\theta h(\theta)-F\left(p_{c}, h(\theta)\right)+F\left(p_{c}, 0\right)=\int_{0}^{h(\theta)} \theta-\frac{\partial F}{\partial h}\left(p_{c}, y\right) d y,
$$


and observing that $y \rightarrow \partial F / \partial h\left(p_{c}, y\right)$ is increasing and $=\theta$ at $y=h(\theta)$, we see that the last expression $\left(=\hat{A}^{*}\left(p_{c}, \theta\right)\right)$ is

$$
\approx \theta h(\theta) \approx \theta^{1+\delta} \approx\left(p-p_{c}\right)^{\beta(1+\delta)}
$$

so we must have $\beta(1+\delta) \geqq 2-\alpha_{2}^{\prime}$, the desired inequality.

\section{Inequalities for $\boldsymbol{\Delta}_{2}$}

In this section we will prove

$$
\begin{gathered}
\Delta_{2} \geqq 2, \\
\Delta_{2}^{\prime} \geqq\left(\beta+\gamma^{\prime}\right) \wedge 2 .
\end{gathered}
$$

The starting point is letting

$$
\chi(p)=E_{p}\left(\left|C_{0}\right| ;\left|C_{0}\right|<\infty\right),
$$

and observing that

$$
\chi(p)=\sum_{n, m} n \alpha_{n, m} p^{n}(1-p)^{m}
$$

so

$$
\left(\frac{d \chi}{d p}\right)^{2}=\left(\sum_{n, m} n\left(\frac{n}{p}-\frac{m}{1-p}\right) \alpha_{n, m} p^{n}(1-p)^{m}\right)^{2},
$$

and using Cauchy-Schwarz gives

$$
\left(\frac{d \chi}{d p}\right)^{2} \leqq\left(\sum_{n, m} n^{2} \alpha_{n, m}(p)\right)\left(\sum_{n, m}\left(\frac{n}{p}-\frac{m}{1-p}\right)^{2} \alpha_{n, m}(p)\right)
$$

where

$$
\alpha_{n, m}(p)=\alpha_{n, m} p^{n}(1-p)^{m}=P_{p}\left(\left|C_{0}\right|=n,\left|\partial C_{0}\right|=m\right) .
$$

The first term on the right-hand side of $(*)$ is $E_{p}\left(\left|C_{0}\right|^{2} ;\left|C_{0}\right|<\infty\right)$. To bound the second we let $M(p)=P_{p}\left(\left|C_{0}\right|=\infty\right)$, and observe that

$$
M(p)=1-\alpha_{0}(p)-\sum_{n, m} \alpha_{n, m} p^{n}(1-p)^{m},
$$

and $1-\alpha_{0}(p)=p$, so differentiating gives

$$
\begin{aligned}
\frac{\partial M}{\partial p}= & 1-\sum_{n, m}\left(\frac{n}{p}-\frac{m}{1-p}\right) \alpha_{n, m} p^{n}(1-p)^{m} \\
\frac{\partial^{2} M}{\partial p^{2}}= & -\sum_{n, m}\left(\frac{n}{p}-\frac{m}{1-p}\right)^{2} \alpha_{n, m} p^{n}(1-p)^{m} \\
& +\sum_{n, m}\left(\frac{n}{p^{2}}+\frac{m}{(1-p)^{2}}\right) \alpha_{n, m} p^{n}(1-p)^{m}
\end{aligned}
$$


If $p<p_{c}$ then $M(p) \equiv 0$ so

$$
\begin{gathered}
\sum_{n, m}\left(\frac{n}{p}-\frac{m}{1-p}\right)^{2} \alpha_{n, m}(p)=\sum_{n, m}\left(\frac{n}{p^{2}}+\frac{m}{(1-p)^{2}}\right) \alpha_{n, m}(p) \\
=E\left(\frac{\left|C_{0}\right|}{p^{2}}+\frac{\left|\partial C_{0}\right|}{(1-p)^{2}}\right) \leqq\left(\frac{1}{p^{2}}+\frac{K}{(1-p)^{2}}\right) E\left|C_{0}\right|
\end{gathered}
$$

where $K=\sup _{x}|\{y:(x, y) \in B\}|$ is the maximum coordination number of the lattice. We have assumed $K<\infty$ and $p_{c} \in(0,1)$, so near $p_{c}$ we have

$$
\left(\frac{d \chi}{d p}\right)^{2} \leqq\left(E\left|C_{0}\right|^{2}\right) K^{\prime} \chi
$$

for some $K^{\prime} \in(0, \infty)$ or

$$
E\left|C_{0}\right|^{2} \geqq \frac{K^{\prime}}{\chi(p)}\left(\frac{d \chi}{d p}\right)^{2}
$$

If $d \chi / d p \approx\left(p-p_{c}\right)^{-\gamma-1}$ then $\chi(p) \approx\left(p-p_{c}\right)^{-\gamma}$ and the right-hand side $\approx\left(p-p_{c}\right)^{-2}$, proving (3).

When $p>p_{c}$, things are a little more complicated. $M(p) \neq 0$ so we have

$$
\sum_{n, m}\left(\frac{n}{p}-\frac{m}{1-p}\right)^{2} \alpha_{n m}(p)=E\left(\frac{\left|C_{0}\right|}{p^{2}}+\frac{\left|\partial C_{0}\right|}{(1-p)^{2}}\right)-\frac{\partial^{2} M}{\partial p^{2}}
$$

Now $\partial M / \partial p \geqq 0$, but nothing is known about the sign of $\partial^{2} M / \partial p^{2}$. In most models we expect that $M$ will be concave near $p_{c}$ (since we expect $\beta \leqq 1$ in general), so we will start with that case.

When $-\partial^{2} M / \partial p^{2}>0$ near $p_{c}$ there is no possibility of cancellation so we have (recall $x^{-2} \geqq x^{-1}$ when $0<x<1$ )

$$
\begin{gathered}
R(p) \equiv E\left(\frac{\left|C_{0}\right|}{p^{2}}+\frac{\left|\partial C_{0}\right|}{1-p^{2}}\right)-\frac{\partial^{2} M}{\partial p^{2}} \approx\left(p-p_{c}\right)^{-\gamma \wedge(\beta-2)} \\
E\left|C_{0}\right|^{2} \geqq\left(\frac{\partial E\left|C_{0}\right|}{\partial p}\right)^{2} R(p)^{-1} \approx\left(p-p_{c}\right)^{2(-\gamma-1)-(-\gamma \wedge(\beta-2))},
\end{gathered}
$$

and hence

$$
\gamma-\Delta_{2}^{\prime} \leqq 2\left(-\gamma^{\prime}-1\right)-\left(-\gamma^{\prime} \wedge(\beta-2)\right)
$$

If $\beta-2 \geqq-\gamma^{\prime}$, the above reduces to $-\Delta_{2}^{\prime} \leqq-2$. If $\beta-2 \leqq-\gamma^{\prime}$, then we get $-\Delta_{2}^{\prime}$ $\leqq-\gamma^{\prime}-\beta$. Combining the two inequalities gives $\Delta_{2}^{\prime} \geqq 2 \wedge\left(\beta+\gamma^{\prime}\right)$, which is (3) when $\partial^{2} M / \partial p^{2}<0$ near $p_{c}$.

If $\partial^{2} M / \partial p^{2}$ is bounded above then the last proof still works, so ignoring pathologies we are left with the case $\partial^{2} M / \partial p^{2} \rightarrow+\infty$ as $p \rightarrow p_{c}$ (which we conjecture never occurs). In this case we can ignore the second term and argue as in the case $p<p_{c}$ to conclude that $\Delta^{\prime} \geqq 2$, which is the desired inequality since $R(p) \geqq 0$ implies $\beta-2 \geqq-\gamma^{\prime}$, i.e. $\beta+\gamma^{\prime} \geqq 2$. 


\section{Appendix. Free Energy for Percolation on the Binary Tree}

In this section we will consider percolation on the binary tree (Example 5 of Sect. 1) and derive an exact expression for the free energy $F(p, h)$ defined in Sect. 2 . The derivation given below is based on arguments of [13]. The starting point is the observation that (in the notation of Sect. 2)

$$
\alpha_{n, m}=0 \text { unless } m=n+1 .
$$

To prove (1) we observe that it is true for $n=1$ and adding a site in $\partial C_{0}$ removes one boundary site but adds two new ones.

With (1) in hand, the problem of calculating $F(p, h)$ becomes greatly simplified because

$$
F(p, h)=h p+\sum_{n=1}^{\infty} n^{-1} \alpha_{n, n+1} p^{n}(1-p)^{n+1} e^{-n h},
$$

and if we let $b_{n}=n^{-1} \alpha_{n, n+1}$ and

$$
B(\theta)=\sum_{n=1}^{\infty} b_{n} \theta^{n}
$$

then we can write

$$
F(p, h)=h p+(1-p) B\left(p(1-p) e^{-h}\right) .
$$

Now if $p<\frac{1}{2}$,

$$
M(p, 0)=\frac{\partial F}{\partial h}(p, 0)=0,
$$

so differentiating (2) with respect to $h$ and setting $h=0$ gives

$$
\begin{gathered}
\frac{\partial F}{\partial h}(p, h)=p+(1-p) B^{\prime}\left(p(1-p) e^{-h}\right)\left(-e^{-h} p(1-p)\right), \\
0=p-p(1-p)^{2} B^{\prime}(p(1-p)),
\end{gathered}
$$

or

$$
B^{\prime}(p(1-p))=1 /(1-p)^{2} .
$$

Now if $p(1-p)=x$, then $p^{2}-p+x=0$, so solving gives

$$
p=\frac{1 \pm \sqrt{1-4 x}}{2} .
$$

The right-hand side is $<1 / 2$ if we pick the solution with - , so substituting this root for $p$ in (3) gives

$$
B^{\prime}(x)=1 /\left(\frac{1+\sqrt{1-4 x}}{2}\right)^{2} x \leqq \frac{1}{4},
$$

and since $B(0)=0$, it follows that

$$
B(0)=\int_{0}^{x} 4 /(1+\sqrt{1-4 y})^{2} d y, \quad x \leqq \frac{1}{4} .
$$


The reader should note that $B^{\prime}(1 / 4)=4$, so $B$ does not $\rightarrow \infty$ at $x=1 / 4$, it just becomes complex for $x>1 / 4$.

Equations (2) and (4) when combined give the formula we gave for $F(p, h)$ in Sect. 3. To verify now that $\partial^{2} F / \partial p^{2}<0$ it remains then to differentiate (2) and evaluate the result,

$$
\begin{gathered}
F(p, 0)=(1-p) B(p(1-p)) \\
\frac{\partial F}{\partial p}(p, 0)=-B(p(1-p))+(1-p) B^{\prime}(p(1-p))(1-2 p) \\
\frac{\partial^{2} F}{\partial p^{2}}(p, 0)=(-(1-2 p)-(1-2 p)-2(1-p)) B^{\prime}(p(1-p)) \\
+(1-p)(1-2 p)^{2} B^{\prime \prime}(p(1-p))
\end{gathered}
$$

The second term $\rightarrow 0$ as $p \rightarrow 1 / 2\left[B^{\prime \prime} \rightarrow \infty\right.$ but $(1-2 p)^{2} \rightarrow 0$ faster $]$, so

$$
\frac{\partial^{2} F}{\partial p^{2}}(1 / 2,0)=-B^{\prime}(1 / 4)=-4,
$$

as we claimed in Sect. 3. The reader should note that differentiating again leads to a term of the form $2(1-p)(1-2 p) B^{\prime \prime}(p(1-p))$, and

$$
B^{\prime \prime}(x)=-2\left(\frac{1+\sqrt{1-4 x}}{2}\right)^{-3} \frac{1}{4} \frac{1}{\sqrt{1-4 x}}(-4),
$$

so $\partial^{3} F / \partial p^{3}$ is discontinuous at $(1 / 2,0)$. This result is, of course, "well known," see [11].

\section{References}

1. Aizenman, M.: Geometric analysis of $\phi^{4}$ fields and Ising models. Parts I and II. Commun. Math. Phys. 86, 1-48 (1982)

2. Aizenman, M., Newman, C.N.: Tree graph inequalities and critical behavior in percolation models. J. Stat. Phys. (to appear 1984)

3. Amit, D.J.: Field theory, the renormalization group, and critical phenomena. New York: McGraw-Hill 1978

4. Athreya, K., Ney, P.: Branching processes. Berlin, Heidelberg, New York: Springer 1972

5. Dhar, D., Barna, M.: Monte Carlo simulation of directed percolation on a square lattice. J. Phys. C. 14, L1-L6 (1981)

6. Domany, E., Kinzel, W.: Directed percolation in two dimensions: numerical analysis and an exact solution. Phys. Rev. Lett. 47, 5-8 (1981)

7. Durrett, R.: Conditioned limit theorems for some null recurrent Markov processes. Ann. Prob. 6, 798-828 (1978)

8. Durrett, R.: Oriented percolation in two dimensions. Ann. Prob. 12, 999-1040 (1984)

9. Durrett, R.: Some general results concerning the critical exponents of percolation processes. ZFW (to appear 1985)

10. Essam, J.W., Gwilym, K.M.: The scaling laws for percolation processes. J. Phys. C 4, L228-232 (1971)

11. Essam, J.W.: Percolation theory. Rep. Prog. Phys. 43, 833-911 (1980)

12. Feller, W.: Theory of probability and its applications, Vol. II. New York: Wiley 1970 
13. Fisher, M.E., Essam, J.W.: Some cluster size and percolation problems. J. Math. Phys. 2, 609-619 (1961)

14. Fisher, M.E.: The theory of equilibrium critical phenomena. Rep. Prog. Phys. 30, 615-730 (1967)

15. Griffiths, R.B.: Ferromagnets and simple fluids near the critical point: Some thermodynamic inequalities. J. Chem. Phys. 43, 1958-1968 (1965)

16. Grimmett, G.R.: On the differentiability of the number of clusters per vertex in the percolation model. J. London Math. Soc. 23, 372-384 (1981)

17. Kesten, H.: Analyticity properties and power law estimates of functions in percolation theory. J. Stat. Phys. 25, 717-756 (1981)

18. Kesten, H.: Percolation theory for mathematicians. Boston: Birkhäuser 1982

19. Kunz, H., Soulliard, B.: Essential singularity in percolation problems and asymptotic behavior of cluster size distributions. J. Stat. Phys. 19, 77-106 (1978)

20. Nienhuis, B., Riedel, E.K., Schick, M.: Magnetic exponents of the two dimensional $q$-state Potts model. J. Phys. A 13, L189-L192 (1980)

21. Rushbrooke, G.S.: On the thermodynamics of the critical region for the Ising problem. J. Chem. Phys. 39, 842-843 (1963)

22. Sokal, A.: More inequalities for critical exponents. J. Stat. Phys. 25, 25-51 (1981)

23. Stanley, H.E.: Introduction to phase transitions and critical phenomena. Oxford: Oxford University Press 1971

24. Stoer, J., Witzgall, C.: Convexity and optimization in finite dimensions. Berlin, Heidelberg, New York: Springer 1970

25. Wu, F.Y.: Percolation and the Potts model. J. Stat. Phys. 18, 115-123 (1978)

26. Wu, F.Y.: Domany-Kinzel model of directed percolation: formulation as a random walk problem and some exact results. Phys. Rev. Lett. 48, 775-778 (1982)

Communicated by J. Fröhlich

Received September 28, 1984 
